\title{
Gestión estratégica del recurso pluvial urbano: condición actual en Colombia*
}

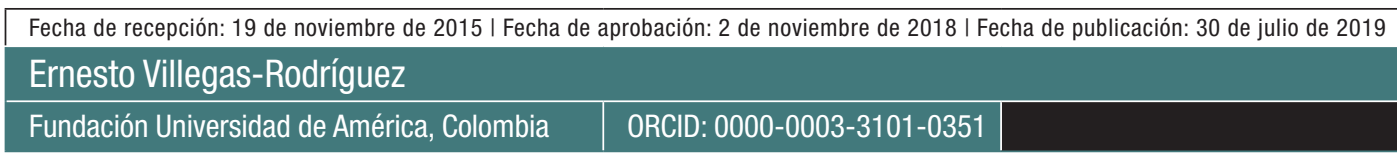

\begin{tabular}{l} 
Gustavo Sandoval-Betancour \\
\hline Fundación Universidad de América, Colombia
\end{tabular}

\begin{tabular}{|c|c|}
\hline Ivia Isabel Casas-Matiz & \\
\hline Universidad Católica de Colombia, Colombia & ORCID: 0000-0002-8200-6697 \\
\hline
\end{tabular}

\begin{tabular}{|c|c|}
\hline Universidad Piloto de Colombia, Colombia & ORCID: 0000-0002-8836-2916 \\
\hline
\end{tabular}

Luis Fernando Molina-Prieto

Resumen El artículo evalúa las condiciones actuales que definen la gestión del recurso pluvial urbano en Colombia. Se presenta un panorama internacional de los nuevos paradigmas para la gestión del recurso pluvial urbano, los principales factores que generan inundaciones en el territorio colombiano, un análisis comparado entre las normativas urbanas de cinco países europeos y las vigentes hoy día en Colombia, los factores primordiales a tener en cuenta para diseñar arquitectura sensible al agua, y los beneficios económicos de gestionar el recurso pluvial en Bogotá, de manera que la ciudadanía y la ciudad se beneficien técnica y económicamente de la gestión adecuada del recurso. Se concluye que en Colombia son incipientes las políticas públicas que tienen en cuenta las aguas lluvias, y que en el campo de la planificación aún no existe un proyecto que las integre como parte del diseño urbano o el paisaje de la ciudad colombiana contemporánea.

Palabras clave resiliencia urbana, control de inundaciones, cambio climático

\footnotetext{
Artículo de investigación científica y tecnológica

Este artículo es producto del trabajo de investigación "Gestión del recurso pluvial y su uso potencial en la planeación del territorio" financiado por la Fundación Universidad de América, sede Bogotá.
} 


\section{Strategic Management of the Urban Rainwater Resource:}

Current Conditions in Colombia

Abstract This article evaluates the current conditions defining the management of the urban rainwater resource in Colombia. An international overview of the new paradigms for the urban rainwater resource management is provided, as well as the main factors causing floods in the Colombian territory. This work also provides a comparative analysis of the urban rules/regulations in five European countries and those currently in force in Colombia, and the primary factor to be considered when designing water-sensitive architectures with the financial benefits derived from the rainwater resource management in Bogotá. The aim is to bring technical and financial benefits to both the city and citizen. It is concluded that in Colombia there are poor public policies taking into account the rainwater. Likewise, in the planning efforts there is not yet any project integrating the rainwater as a part of the urban design or the urban landscape in the contemporary Colombian cities.

Keywords urban resilience, flooding control, climate change

\section{Gestão estratégica do recurso pluvial urbano: condição atual na Colômbia}

Resumo $\mathrm{O}$ artigo avalia as condiçóes atuais que definem a gestão do recurso pluvial urbano na Colômbia. Apresenta-se um panorama internacional dos novos paradigmas para a gestão do recurso pluvial urbano, os principais fatores que geram inundações no território colombiano, uma análise comparada entre a regulamentação urbana de cinco países europeus e em vigor hoje na Colômbia, os fatores primordiais a levar em conta para desenhar arquitetura sensível à agua, e os benefícios econômicos de gerir o recurso pluvial em Bogotá, de maneira a beneficiar técnica e economicamente a cidadania e a cidade de isso. Conclui-se que na Colômbia são incipientes as políticas públicas que levam em conta as aguas-chuva e que no campo do planejamento ainda náo existe um projeto que as integre como parte do desenho urbano ou a paisagem da cidade colombiana contemporânea.

Palavras chave resiliência urbana, controle de inundações, mudança climática 


\section{Introducción}

El acceso al agua dulce en las ciudades es cada día más limitado. El crecimiento demográfico y las migraciones campo-ciudad, la destrucción de los manantiales y la polución de los cuerpos de agua, el acaparamiento del agua con ánimo de lucro y las elevadas tarifas de los acueductos, junto con el enorme despilfarro del agua potable ${ }^{1}$ son algunos de los factores que incrementan el desabastecimiento de agua en la actualidad (Buhaug y Urdal, 2013; Fernández-Pérez, 2009; Annecchini, 2005). Otro factor de gran relevancia es el cambio climático, fenómeno actualmente aceptado por la comunidad científica que genera dos situaciones en relación con las aguas urbanas, por un lado, rigurosas sequías; y por otro, inundaciones incontrolables, como las acontecidas recientemente en Suecia (Göransson, 2013), Australia (Wilby y Keenan, 2012), India (Ranger et al., 2011) o Colombia (Carvajal-Escobar, 2011), por solo citar algunos de esos catastróficos eventos en diversas regiones del planeta.

La creciente necesidad de agua potable sumada a su gestión actual, que se puede calificar como insostenible en muchos países y ciudades del mundo ${ }^{2}$, no solo genera competencia por el recurso hídrico, sino que ha desencadenado verdaderas luchas, conflictos y guerras en todo el orbe. Shiva (2004) considera que los conflictos por el agua ocurridos en 1924, entre los habitantes de Los Ángeles y los del Valle de Owens en California, fueron el punto de partida de las guerras por el agua; y reseña conflictos por este recurso esencial para la vida en la India, Siria, Irak, Turquía, Anatolia, Egipto, Sudán, Etiopía, Israel,
Jordania, El Líbano y Cisjordania. Bloch (2003) establece tres relaciones básicas: agua-desarrollo urbano, agua-población y agua-pobreza, y estudia una serie de conflictos armados por el agua potable. Gleick (2010) analiza las guerras por el agua en el Medio Oriente y subraya la importancia que el agua dulce tiene como base para la paz en esa región del mundo azotada por la violencia. Otros investigadores estudian conflictos sociales y urbanos por los costos o el acaparamiento del agua potable en diversas ciudades, como México D. F., Cochabamba o Barcelona (Perló-Cohen y González-Reynoso, 2009; Kruse, 2005; Tello-Aragay, 1998).

Frente a la creciente necesidad de agua potable y el riesgo de inundación que amenaza a las urbes contemporáneas, se empiezan a vislumbrar nuevos paradigmas para su gestión en las ciudades. Nelson, Moddemeyer y Schwartz (2007) subrayan que las tradicionales infraestructuras hidráulicas urbanas -el acueducto junto con el sumidero y las tuberías subterráneas del alcantarillado- protegieron en el pasado a los ciudadanos de sequías, inundaciones y enfermedades, pero ahora son sistemas obsoletos que ponen en peligro tanto a las ciudades como a sus habitantes. Por su parte, Bacchin, Ashley, Sijmons, Zevenbergen y Timmeren (2014) señalan que las infraestructuras para la gestión del agua en las ciudades se han concebido históricamente de manera independiente y separada del diseño y la planificación urbana; se han construido en el subsuelo, ocultas y desvinculadas del paisaje urbano y, por ende, en muchas ocasiones generan 
incompatibilidades con el contexto urbano. Es necesario, por tanto, generar nuevos paradigmas para la gestión del recurso pluvial, que superen y dejen atrás el sumidero tradicional y la tubería subterránea, hoy en día obsoletas e inoperantes en gran parte de las ciudades. Estos sistemas -por no ser fáciles de adecuar, ensanchar o reemplazar al ritmo creciente de la población- transforman a las ciudades en sistemas vulnerables que, frente a eventos de poca magnitud, como una tormenta prolongada, sufren colapsos urbanos perturbadores con catastróficas consecuencias para la población, la ciudad y su economía (Adger, 2006). En consecuencia, es urgente generar en Colombia políticas y estrategias, especialmente, normativas urbanas, que involucren el agua lluvia en la planificación urbana para que el diseño urbano se trasforme en un ejercicio que genere ciudades sustentables y resilientes.

Teniendo en cuenta todo lo anterior, el artículo examina el modelo actual de gestión del recurso pluvial en Colombia, haciendo énfasis en Bogotá, con el fin de valorar el estado en que se encuentran a este respecto las políticas que rigen la gestión pública (decretos, acuerdos, leyes y normativas). Se analizan los criterios generales para la gestión del recurso pluvial desde el objeto arquitectónico, se presentan algunas iniciativas surgidas desde las instancias privadas, y se analizan las propuestas e investigaciones desarrolladas desde la academia. Finalmente, se exponen algunas ventajas económicas de acumular y aprovechar las aguas lluvias a nivel urbano y arquitectónico.

\section{Resultados}

Gestión urbana del recurso pluvial: panorama internacional

En Estados Unidos, Canadá, Australia y algunos países europeos se han desarrollado en la última década nuevas estrategias para la gestión de las aguas pluviales. Se trata de nuevos paradigmas que aprovechan el recurso pluvial para la ciudad y para la arquitectura, y al hacerlo, minimizan, reducen e incluso eliminan los riesgos de inundación en las ciudades. En Estados Unidos sobresalen los diseńos y normativas de la ciudad de Filadelfia, definidos en Green Streets Design Manual of Philadelphia (Philadelphia Water Department, 2014), manual técnico que establece de manera detallada la gestión de las aguas pluviales en las áreas urbanas. En Canadá, llama la atención los estructurados lineamientos para la gestión de las aguas pluviales desarrollados para la región de Quebec, que se presentan en los 14 capítulos de la Guide de gestion des eaux pluviales (Environnement et Lutte contre les changements climatiques Québec, 2014). En esta guía técnica se abordan cuatro escalas para la gestión del recurso pluvial: la planificación de las cuencas del territorio, la planificación de las sub-cuencas rurales y urbanas, la planificación de las cuencas urbanas de drenaje, y por último, la planificación del lugar de intervención. En Quebec se busca reducir al mínimo los impactos hidrológicos de la urbanización, mediante la flexibilidad, pues la guía presenta diversos enfoques para que los actores involucrados en el desarrollo urbano (urbanistas, arquitectos paisajistas, especialistas ambientales, desarrolladores y responsables municipales) apliquen los criterios técnicos recomendados a partir de su adecuación a cada situación específica.

Australia se destaca por su innovadora propuesta de Water Sensitive Cities (ciudades sensibles al agua), que forma parte de los objetivos del gobierno federal y los gobiernos estatales de ese país. Este modelo de gestión hídrica bien puede ser el estado ideal de las ciudades en cuanto a habitabilidad, productividad, sustentabilidad y resiliencia en el tema de las aguas urbanas, porque rescata y fortalece el ciclo natural del agua en las ciudades, aprovecha recursos hídricos de diversas fuentes, protege la salud de los cuerpos de agua y de 
la biodiversidad que depende de ellos; y crea espacios públicos a cielo abierto que acopian, purifican y hacen uso de las aguas pluviales, de modo que aportan belleza y serenidad al paisaje urbano, reducen el fenómeno de islas de calor y colaboran con el secuestro de carbono (Wong et al., 2013). Por transformar la visión tradicional del diseño urbano, proponiendo armonía y sinergia entre la planificación hidrológica y el ordenamiento urbano, la Water Sensitive City ha desbordado los límites de su país de origen y actualmente sus postulados se estudian y aplican en ciudades de Alemania, Dinamarca, Holanda, Italia, Polonia, Reino Unido, Corea del Sur, Japón, Sur África y Estados Unidos (Boroomand, 2015; Kim, Kwack, Yoon, Lee y Hwang, 2014; Morgan et al., 2013).

En Europa, cinco países están a la vanguardia en el campo de la gestión de las aguas pluviales: Dinamarca, Suecia, Bélgica, Suiza y Francia (en la región del Grand Lyon), puesto que han establecido estrategias o normativas claras y precisas en cuanto a la gestión de las aguas pluviales urbanas que inciden en el diseño urbano. En estos países, al igual que en Australia, se está gestando un gran cambio de paradigma: el agua lluvia ya no se esconde -entubándola en el subsuelo- ni se evacúa del perímetro urbano con urgencia; por el contrario, se ralentiza su circulación por la ciudad por medio de espacios a cielo abierto que se transforman en elementos clave para diseños urbanos polifuncionales, lúdicos, estéticos y amables, que, además, fortalecen la resiliencia hidráulica de las ciudades. En el apartado siguiente se desglosan las más destacadas políticas del agua de estos cinco países europeos y se toman como base para analizar la condición colombiana.

\section{Gestión urbana del recurso pluvial:} la planificación territorial

Un factor que contribuye notoriamente a las inundaciones urbanas en Colombia es el inadecuado proceso de ordenamiento y planificación, que altera el paisaje sin tener en cuenta los sistemas de drenaje natural del territorio. Ejemplo de lo anterior fue la urbanización de la Sabana de Bogotá durante el siglo XX, pues en su esfuerzo por satisfacer la enorme necesidad de hábitat que requería la creciente población de la ciudad de Bogotá, generó incontables desarrollos urbanos sobre el sistema de lagos y humedales ${ }^{3}$ asociados al río Bogotá y sus afluentes. De esta forma, y por cuenta de la urbanización, se destruyó el sistema natural que controlaba las inundaciones, puesto que durante las temporadas lluviosas los lagos y humedales actuaban como amortiguadores naturales de las crecientes y los desbordamientos, y, además, regulaban el ciclo hidrológico, puesto que permitían la recarga de los acuíferos, una función que se obstaculizó por completo con la urbanización, debido a que las construcciones, andenes y asfaltos impermeabilizan la mayor parte del suelo (Departamento Técnico Administrativo del Medio Ambiente [DAMA], 2000). En consecuencia, muchos barrios construidos sobre los humedales, como Patio Bonito, por solo mencionar un ejemplo, sufrieron constantes inundaciones desde su inauguración hasta que se construyó el jarillón que retiene los desbordamientos naturales del río Bogotá en el área que ocupa dicho barrio (que originalmente formaba parte del humedal de La Vaca). Este factor antrópico de inundación, la planificación que desconoce los ciclos naturales del territorio, conlleva enormes impactos en muchas áreas urbanas del país, precisamente donde se concentra la mayor cantidad de habitantes e infraestructura, tanto económica como sociocultural. Por tanto, las consecuencias de las inundaciones son más graves y profundas para la sociedad cuando acontecen en las ciudades.

Cabe subrayar que la topografía colombiana juega un papel fundamental en la gestión de las aguas pluviales, especialmente en las regiones andinas, donde se concentra la mayor parte de 
las ciudades y el mayor número de habitantes. Desafortunadamente, este factor no es tenido en cuenta en profundidad, de manera que, en la actualidad, en muchas ciudades y municipios se permite la construcción de barrios enteros en zonas de ladera, lo cual representa un alto riesgo de deslave. Además, muchos de esos barrios, durante las temporadas de lluvia intensa, sufren deslizamientos y avalanchas catastróficas, que en ocasiones conllevan consecuencias funestas. Estas son tragedias anunciadas que bien se podrían evitar, siempre y cuando se pusiera mayor atención a las características naturales (agua, suelo, topografía, vegetación, etc.) del territorio al momento de definir las políticas públicas y las normativas para la planificación y el desarrollo de los centros urbanos.

Un tercer factor que favorece las inundaciones en municipios y ciudades colombianas es la extracción desordenada de los recursos naturales, que no obedece a una planificación inteligente o previsiva sino a intereses particulares, altera irreversiblemente el paisaje natural, y degrada o destruye los ecosistemas. A este respecto es importante subrayar tres elementos que afectan los escenarios naturales y transforman a las ciudades en sistemas vulnerables frente a las lluvias, aun siendo leves: i) deforestación de las cabeceras de los ríos y las áreas ribereñas, que favorecen la formación de grandes e inesperadas crecientes; ii) minería a cielo abierto, que destruye los cauces naturales de las aguas superficiales (desde escorrentías hasta grandes ríos), lo que genera un fuerte desorden hidrológico; y iii) ocupación indebida de rondas de ríos, quebradas y humedales, que altera el funcionamiento natural de los ecosistemas hídricos y, en muchos casos, es el primer paso para la desecación de los cuerpos de agua (especialmente de lagos y humedales).

\section{Gestión urbana del recurso pluvial:}

\section{la política urbana}

De igual manera, y como parte integral de la investigación, se llevó a cabo una revisión de documentos oficiales enfocados en la gestión estratégica del recurso pluvial urbano. Se seleccionaron cinco países europeos por estar a la vanguardia en ese campo: Dinamarca, Suecia, Bélgica, Suiza y Francia. La tabla 1 presenta una síntesis de las políticas, estrategias, leyes y normativas urbanas relacionadas con la gestión del recurso pluvial en esos cinco países, y, finalmente, los resultados obtenidos en el mismo sentido para Colombia.

Tabla 1. Formas de gestión del agua Iluvia. Revisión comparada Europa-Colombia

\begin{tabular}{|c|c|c|}
\hline & Política nacional & Política local \\
\hline & Existencia y evidencia & Existencia y evidencia \\
\hline \multirow{4}{*}{ 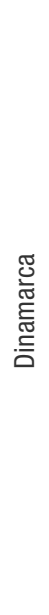 } & $\begin{array}{l}\text { Manual Uso del agua Iluvia (Miljøministeriet, 2014), publicado } \\
\text { por la Agencia de Protección del Medio Ambiente, la Agencia } \\
\text { Danesa de Energía y el Ministerio de Medio Ambiente. Documento } \\
\text { respaldado por la Ley del Agua de } 2000 .\end{array}$ & $\begin{array}{l}\text { Planes estratégicos para la sostenibilidad como el de Aalborg } \\
\text { (2013), estrategias para el manejo de las aguas subterráneas } \\
\text { como la de Allerød (2012), estrategias para la gestión del agua } \\
\text { Iluvia como la de Furesø (2010); estrategias de adaptación al } \\
\text { cambio climático como las de Copenhague (Københavns, 2011), } \\
\text { Hvidovre (2014), Roskilde (2013), Odense (2011), Ringsted (2011) } \\
\text { y Gentofte (2010). }\end{array}$ \\
\hline & $\begin{array}{l}\text { Plan de acción para la protección contra el clima en Dinamarca } \\
\text { (Naturstyrelsen, 2012/2013) publicado por la Agencia para la } \\
\text { Naturaleza y el Grupo de Trabajo para la Adaptación al Cambio } \\
\text { Climático. }\end{array}$ & $\begin{array}{l}\text { Objetivos: Fortalecer la sustentabilidad. Fortalecer la resiliencia } \\
\text { urbana. Evitar inundaciones. Acopiar agua lluvia. }\end{array}$ \\
\hline & $\begin{array}{l}\text { Objetivos: evitar inundaciones por tormentas urbanas. } \\
\text { Acopiar agua lluvia para fines sanitarios. }\end{array}$ & Criterios de uso del agua lluvia: lúdica, paisajismo, ingeniería. \\
\hline & Criterios de uso del agua lluvia: lúdica, paisajismo, ingeniería. & $\begin{array}{l}\text { Incentivos: subsidios a proyectos que incluyan la gestión del agua } \\
\text { Iluvia en su diseño. }\end{array}$ \\
\hline
\end{tabular}




\begin{tabular}{|c|c|c|}
\hline & Política nacional & Política local \\
\hline & Existencia y evidencia & Existencia y evidencia \\
\hline \multirow{4}{*}{$\begin{array}{l}\frac{\pi}{0} \\
\frac{\mathbb{d}}{3}\end{array}$} & $\begin{array}{l}\text { Manual para la gestión de aguas pluviales en viaductos } \\
\text { (Trafikverket, 2011), publicado por la Dirección General de } \\
\text { Carreteras y la Agencia para el Medio Ambiente. }\end{array}$ & $\begin{array}{l}\text { Manual Cuida tu agua (Stockholms Stad, 2001), que establece } \\
\text { la 'disposición local de aguas lluvias-LOD' en Estocolmo; } \\
\text { y Estrategia de Aguas Pluviales de la Ciudad de Estocolmo } \\
\text { (Stockholms Stad, 2013). }\end{array}$ \\
\hline & $\begin{array}{l}\text { Agencia sueca de protección ambienta/ (Naturvårdsverket, 2019). } \\
\text { Objetivos para la gestión de las aguas pluviales. }\end{array}$ & $\begin{array}{l}\text { Estrategia de Aguas Pluviales de Huddinge (2000); Masthusen } \\
\text { (2013); Kungsbacka (2012); Österåkers (2010); Värmdö (2012); } \\
\text { Botkyrka (2012); Härryda (2011); Nacka (2011); Tyresö (2010); } \\
\text { Skåne (2009) y Falu (2008). }\end{array}$ \\
\hline & $\begin{array}{l}\text { Objetivos: evitar inundaciones por tormentas en viaductos urbanos } \\
\text { y rurales. }\end{array}$ & $\begin{array}{l}\text { Objetivos: gestionar la mayor parte del agua lluvia in situ. Evitar } \\
\text { recarga de alcantarillados y plantas de tratamiento. Suprimir } \\
\text { escorrentías e inundaciones. Mantener nivel freático. Reducir } \\
\text { índice de impermeabilización del suelo. Lograr ciudades más } \\
\text { resilientes. Lograr un paisaje urbano más hermoso. Crear techos } \\
\text { verdes. Instalar biorretenedores. }\end{array}$ \\
\hline & $\begin{array}{l}\text { Criterios de uso del agua lluvia: conducción de manera superficial } \\
\text { de aguas pluviales; rescatar, restaurar e incluso crear cursos de } \\
\text { agua en las ciudades. }\end{array}$ & $\begin{array}{l}\text { Criterios de uso del agua lluvia: Elemento para el diseño y la } \\
\text { planificación urbana (conducción superficial). }\end{array}$ \\
\hline \multirow{3}{*}{ 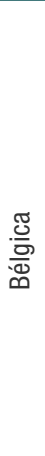 } & $\begin{array}{l}\text { Normativa urbana. Reglamento Regional de Urbanismo, Artículo } \\
\text { 16: "Colecta de aguas pluviales: La escorrentía de aguas pluviales } \\
\text { generada por todas las superficies impermeables debe ser } \\
\text { recogida y llevada a un tanque [...] El tamaño mínimo de este } \\
\text { tanque es de } 33 \text { litros por } \mathrm{m}^{2} \text { de superficie de cubierta" (BUP } \\
\text { Bruxelles Urbanisme et Patrimoine, 2006, p. 21). }\end{array}$ & $\begin{array}{l}\text { Ficha EAU01 expedida por el Instituto para la Gestión del Ambiente } \\
\text { (IBGE, 2010). }\end{array}$ \\
\hline & $\begin{array}{l}\text { Objetivos: reducir escorrentías a cero. Impedir formación de } \\
\text { inundaciones urbanas. Acopiar agua lluvia para diversos usos. } \\
\text { Gestionar agua pluvial in situ. }\end{array}$ & $\begin{array}{l}\text { Objetivos: gestionar el agua pluvial in situ. Reducir índice de } \\
\text { impermeabilización del suelo. Fortalecer ciclo del agua. Reducir } \\
\text { escorrentías urbanas. Recolectar aguas pluviales para diversos } \\
\text { usos. Instalar techos verdes y sistemas de biorretención. }\end{array}$ \\
\hline & $\begin{array}{l}\text { Criterios de uso del agua lluvia: seguridad, resiliencia, } \\
\text { abastecimiento. }\end{array}$ & $\begin{array}{l}\text { Criterios de uso del agua lluvia: seguridad, resiliencia, } \\
\text { abastecimiento. }\end{array}$ \\
\hline \multirow{5}{*}{ 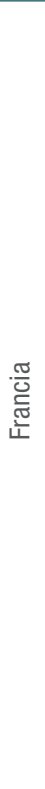 } & Código General del Territorio, Artículo L2224-10 (Legifrance, 2014). & Normativa en la región del Grand Lyon \\
\hline & Código Civil, artículos 640, 641 y 681. & $\begin{array}{l}\text { Gestión integrada de las aguas pluviales. Naturaleza y bienestar } \\
\text { en la ciudad: Soluciones adaptadas al cambio climático (Grand } \\
\text { Lyon, 2013). }\end{array}$ \\
\hline & $\begin{array}{l}\text { Objetivos: reducir impermeabilización del suelo urbano. Gestionar } \\
\text { agua pluvial in situ. }\end{array}$ & $\begin{array}{l}\text { Las obras aéreas para la gestión de las aguas pluviales. Diseño y } \\
\text { gestión referencial de espacios públicos (Grand Lyon, 2010). }\end{array}$ \\
\hline & Criterios de uso del agua lluvia: seguridad, resiliencia. & $\begin{array}{l}\text { Criterios de uso del agua lluvia: i) lluvia determinante para el } \\
\text { desarrollo urbano; ii) elemento de diseño para la recreación } \\
\text { urbana y el paisajismo; iii) clave para la conservación de } \\
\text { los ecosistemas y la biodiversidad en ambientes urbanos y } \\
\text { periurbanos; iv) patrimonio ciudadano; v) elemento ordenador en } \\
\text { centros urbanos experimentales o innovadores; vi) reductor del } \\
\text { consumo de agua potable en las ciudades; y vii) parte del proyecto } \\
\text { europeo Aqua-ADD. }\end{array}$ \\
\hline & $\begin{array}{l}\text { Incentivos fiscales: tarifas de deducción de impuestos asociadas } \\
\text { a la gestión de aguas pluviales, establecidas en } 2012 \text { por el } \\
\text { Ministerio de Ecología. }\end{array}$ & \\
\hline
\end{tabular}




\begin{tabular}{|c|c|c|}
\hline & Política nacional & Política local \\
\hline & Existencia y evidencia & Existencia y evidencia \\
\hline \multirow{6}{*}{$\stackrel{\mathbb{N}}{\overline{5}}$} & Legislación vigente. & Normativa en el Cantón de Ginebra. \\
\hline & $\begin{array}{l}\text { Ley de Aguas, artículos } 54 \text { y } 64 \text { (Site officiel de l'Etat de Genève, } \\
\text { 2014). }\end{array}$ & $\begin{array}{l}\text { Formulario: Tratamiento de agua en obras de construcción - } \\
\text { Formularie: Traitement des eaux de chantier (Office cantonal de } \\
\text { l'eau [OCEau], 2015). }\end{array}$ \\
\hline & Ley Federal de Protección del Agua (Conseil Fédéral Suisse, 2014). & $\begin{array}{l}\text { Gestión de aguas no contaminadas en la parcela - Aspectos } \\
\text { cuantitativos (Service de la planification de l'eau, 2009). }\end{array}$ \\
\hline & $\begin{array}{l}\text { Ordenanza para la Protección del Agua (Conseil Fédéral Suisse, } \\
\text { 2014). }\end{array}$ & $\begin{array}{l}\text { Objetivos: gestionar el agua pluvial in situ. Incrementar suelo } \\
\text { urbano permeable. Fortalecer ciclo del agua. Reducir escorrentías } \\
\text { urbanas. Recolectar aguas pluviales para diversos usos. Limitar } \\
\text { uso de agua para la construcción. }\end{array}$ \\
\hline & $\begin{array}{l}\text { Objetivos: conservación o rehabilitación del ciclo natural del agua } \\
\text { en áreas urbanas. Gestionar agua pluvial in situ. Reducir daños } \\
\text { por inundaciones. Integrar aguas pluviales a diseño del paisaje } \\
\text { urbano. Integrar aguas pluviales a diseño del paisaje urbano. }\end{array}$ & Criterios de uso del agua lluvia: resiliencia, ciclo del agua. \\
\hline & Criterios de uso del agua lluvia: resiliencia, ciclo del agua. & \\
\hline \multirow{3}{*}{$\frac{\frac{\pi}{0}}{E}$} & $\begin{array}{l}\text { A nivel nacional, el interés por el recurso pluvial es totalmente } \\
\text { incipiente en la política actual, al punto que solamente en dos } \\
\text { documentos se hace referencia a su gestión, en el Decreto } 770 \text { de } \\
214 \text { y en la Resolución } 1096 \text { del } 17 \text { de noviembre de } 2000 \text {. }\end{array}$ & $\begin{array}{l}\text { Normativa para Bogotá: Resolución } 6523 \text { de 2011; Decreto } 528 \\
\text { de } 2014 \text {. }\end{array}$ \\
\hline & $\begin{array}{l}\text { Objetivos: conducir el agua pluvial. Mantener y construir canales } \\
\text { de agua lluvia. Actualizar redes de aguas Iluvias. }\end{array}$ & $\begin{array}{l}\text { Objetivos: reglamentar y adoptar los sistemas urbanos de drenaje } \\
\text { sostenibles SUSD para el plan de ordenamiento zonal norte POZN. } \\
\text { Establecer el Sistema de Drenaje Pluvial Sostenible del Distrito } \\
\text { Capital (SUSD). }\end{array}$ \\
\hline & Criterios de uso del agua lluvia: no existen criterios definidos. & $\begin{array}{l}\text { Criterios de uso del agua lluvia: reducción de riesgos de } \\
\text { inundación, acopio para emergencias, ciclo del agua. }\end{array}$ \\
\hline
\end{tabular}

Fuente: elaboración propia a partir de Miljøministeriet (2014), Naturstyrelsen (2012/2013), Andersen y Steffensen (2011), Aalborg Kommune (2013), Allerød Kommune (2013), Furesø Kommune (2010), Københavns (2011), Hvidovre Kommune (2014), Roskilde Kommune (2013), Odense Kommune (2011), Ringsted Kommune (2011), Gentofte Kommune (2010), Trafikverket (2011), Naturvårdsverket (2019), Stockholms Stad (2001, 2013), Huddinge Kommun (2000), Masthusen (2013), Structor (2016), Kungsbacka Kommun (2012), Österåkers Kommun (2010), Värmdö kommun (2012), Botkyrka Kommun (2012), Härryda Kommun (2011), Nacka Kommun (2011), Tyresö kommun (2010), Skåne Län (2009), Falu Kommun (2008), BUP Bruxelles Urbanisme et Patrimoine (2006), Molina-Prieto (2015), IBGE (2010), Legifrance (2014), Grand Lyon (2010, 2013), Site officiel de l'Etat de Genève (2014), Conseil Fédéral Suisse (2014), Office cantonal de l'eau (OCEau) (2015), Service de la planification de l'eau (2009), Secretaría Distrital de Ambiente (SDA) (2011) y Alcaldía Mayor de Bogotá (2014)

En el tabla 1 se evidencia que mientras en los cinco países tomados como referencia, actualmente la gestión de las aguas pluviales se plantea como elemento clave para: i) la planificación y el diseño de ciudades resilientes y sustentables; ii) el desarrollo del paisaje urbano en armonía con los ciclos naturales; y iii) el aprovechamiento del recurso pluvial por parte de los ciudadanos y las comunidades, además de otros objetivos específicos ${ }^{4}$, el recurso pluvial en Colombia apenas empieza a ser valorado y vinculado a planes, programas y estrategias. Cabe subrayar que esas incipientes iniciativas han surgido muy recientemente, únicamente en Bogotá, y aún no tienen aplicación en la ciudad real.

A nivel nacional, la legislación, la normativa urbana y las políticas públicas (estrategias y planes vigentes en Colombia) no hacen mención al manejo del agua lluvia. En general, el tema de la gestión del agua se mantiene en el paradigma del siglo pasado, es decir, se destaca en todos los 
documentos su importancia en cuanto a abastecimiento y manejo sostenible, determinando únicamente mecanismos de control respecto a concesión de aguas, reglamentación de corrientes y permiso de vertimientos, todo ello sustentado en la noción de agua como bien de uso público 5 .

Gestión urbana del recurso pluvial:

el componente arquitectónico

El objetivo de la gestión del recurso pluvial a nivel arquitectónico es minimizar el impacto por inundaciones en la estructura urbana, la ciudad y el territorio; $y$, de ser posible, acumular la mayor cantidad de agua para aprovecharla en actividades domésticas y de producción, o reintegrarla al ciclo natural del agua. Como criterio general de diseño para la creación de una arquitectura sensible al agua, se debe considerar la gestión eficiente del 100\% del agua lluvia que se precipita sobre el predio, con el fin de evitar la generación de escorrentías en las áreas urbanas y, con ello, las inundaciones. Esta gestión debe incluir la acumulación de agua lluvia en tanques, especialmente diseñados para ese propósito, que estén integrados en la edificación, lo que incrementará la resiliencia de la arquitectura y de la ciudad, al tiempo que reducirá el impacto que el cambio climático pueda tener en relación con el suministro de agua en las ciudades (Aladenola y Adeboye, 2010). El agua lluvia se puede captar mediante diversas superficies (cubiertas, zonas de parqueo, plazoletas, entre otras), pero se debe tener en cuenta que el material de acabado de la superficie de captación incide en la calidad del agua recolectada. Las investigaciones de Mendez et al. (2010), por ejemplo, concluyen que las superficies de metal galvanizado son las que garantizan una mejor calidad del agua captada.

En Colombia son muy pocos los edificios que aprovechan las aguas pluviales. En Bogotá, en el sector público, se destacan dos edificaciones. De un lado, está el Complejo Acuático Simón Bolívar, obra de carácter deportivo, donde la cubierta capta el agua lluvia, la conduce a un tanque de purificación y, tras ser procesada, pasa a un tanque de aguas tratadas en el cual un sistema especializado verifica su saneamiento. Finalmente, se inyecta al sistema hidráulico para labores de limpieza y adecuación de baños y jardines. De otro, se encuentra una construcción de carácter educativo, el Edificio de Postgrados de Ciencias Humanas de la Universidad Nacional de Colombia. La cubierta, transitable y protegida con grava en algunos sectores, capta el agua lluvia; luego, es llevada a tanques subterráneos desde donde se bombea para la descarga de sanitarios (también es usada en las fuentes y los espejos de agua del edificio).

En el sector privado, también en Bogotá, sobresalen dos centros comerciales, Titán Plaza y Unicentro; ambos utilizan el agua lluvia captada en las cubiertas para la descarga de los sanitarios. De igual manera, Alkosto, una empresa comercializadora que cuenta con grandes superficies en Bogotá y Villavicencio, ha implementado la gestión de las aguas pluviales. En la edificación del barrio Venecia, en Bogotá, la cubierta que cuenta con un área de $6000 \mathrm{~m}^{2}$, capta alrededor de $6000 \mathrm{~m}^{3}$ de agua por año. El agua acopiada es tratada en una planta con capacidad para almacenar $40 \mathrm{~m}^{3}$ por día, para después ser inyectada en el sistema hidráulico del edificio. En la edificación de Villavicencio, la cubierta, que cuenta con un área de $1061 \mathrm{~m}^{2}$, capta agua lluvia para ser almacenada en un tanque de $150 \mathrm{~m}^{3}$. Posteriormente, el líquido es tratado por medio de procesos de floculación, filtrado y cloración en una planta de tratamiento. El resultado es agua potable para todas las necesidades del establecimiento durante el año. 
Otra importante opción para la gestión del recurso pluvial en las edificaciones son las cubiertas verdes, sistemas que se clasifican dentro de los biorretenedores (de agua), y que actualmente están siendo testeados en Bogotá, pues forman parte de un proyecto de Paraderos Verdes, sobre la carrera séptima, que busca valorar su comportamiento y eficiencia en las condiciones locales. De igual manera, para fortalecer el uso de techos verdes en la ciudad, en 2014 la Secretaría Distrital de Ambiente publicó Techos Verdes y Jardines Verticales, una guía práctica que incluye los requerimientos mínimos para la implementación de un techo verde y un listado de 78 plantas sugeridas, algunas de las cuales forman parte de la investigación que se adelanta actualmente en los paraderos verdes de la carrera séptima (SDA, 2014).

Desde la academia se ha trabajado en diversos proyectos de investigación que buscan hacer del agua lluvia un recurso útil para la arquitectura y para la ciudad, además de minimizar los riesgos de inundación. Se destaca la Pontificia Universidad Javeriana, por los trabajos experimentales y de investigación que adelanta en varios frentes: i) el proyecto de investigación Aprovechamiento de aguas lluvias en el campus de la Pontificia Universidad Javeriana, sede Bogotá (PUJB): caracterización y posibles usos, que analiza la viabilidad económica y técnica del aprovechamiento del agua lluvia en el campus y su posible uso en función de su calidad; ii) el artículo Hacia equipamientos urbanos sostenibles: aprovechamiento de aguas lluvias en el campus de la Pontificia Universidad Javeriana en Bogotá (Torres et al., 2012), que presenta algunos de los resultados de ese proyecto de investigación; y iii) varias tesis y artículos que abordan el tema del uso de las aguas pluviales desde diferentes enfoques, de autores como Estupiñán-Perdomo y Zapata-García (2010); Gómez, Galarza-Molina y Torres (2018);
Polanco-Andrade y Sánchez-Vega (2012); Torres et al. (2011); Torres-Murillo (2011), por solo citar algunos de ellos.

Otras universidades de Bogotá que están trabajando en el aprovechamiento de las aguas pluviales, sin pretender ser exhaustivos, son: la Universidad Nacional de Colombia (Buitrago-Medina, 2011), la Universidad del Rosario (Orozco-Roa, 2016) y la Universidad de América (Molina-Prieto, 2014, 2015, 2016; Sandoval, 2016). Desafortunadamente, aunque existe un corpus investigativo amplio y consistente, la administración de la ciudad no ha permitido, hasta el momento, que los resultados de investigación en el campo del aprovechamiento de las aguas pluviales permeen las políticas públicas ni las normativas urbanas de la ciudad.

\section{Gestión urbana del recurso pluvial:}

\section{el factor económico}

A partir del Índice de Uso del Agua (IUA) ${ }^{6}$, el Índice de Retención y Regulación Hídrica $(\mathrm{IRH})^{7}$, y el Índice de Vulnerabilidad Hídrica por Desabastecimiento (IVHD) ${ }^{8}$, el Estudio Nacional del Agua (ENA) (Instituto de Hidrología, Meteorología y Estudios Ambientales [IDEAM], 2010), así como el ENA de 2014 (IDEAM, 2014), evidencian que la Sabana de Bogotá es una de las regiones más expuestas a situaciones de alta presión de la demanda sobre la oferta hídrica. Esta situación actual, que exige a la ciudad de Bogotá recurrir al agua dulce de la vertiente del río Orinoco, incrementará con el paso del tiempo. De acuerdo con las proyecciones demográficas y los cálculos de consumo de agua potable para Bogotá en 2035, que se realizaron como parte de la investigación, se evidencia que la población de la ciudad dentro de 20 años rondará los $10 \mathrm{mi}$ llones de habitantes, mientras que el consumo de agua proyectado para ese año, incluyendo los 
consumos residenciales y no residenciales (establecimientos industriales, comerciales, oficiales y especiales) tendrá un incremento porcentual cercano al 37,6\% con respecto al consumo de 2015. Este estimativo se aproxima bastante al orden de magnitud del pronóstico de la Organización de Naciones Unidas en cuanto a consumo de agua mundial para el 2030, que crecería en un $40 \%$ frente al consumo actual (Departamento de Asuntos Económicos y Sociales de Naciones Unidas [ONU-DAES], 2015). Teniendo en cuenta las proyecciones y cálculos anteriores, resulta prioritario para la ciudad de Bogotá establecer políticas, normativas y estrategias que fomenten el uso de fuentes de agua alternativas. Una de esas fuentes, cuya principal ventaja es la de estar disponible para todos los hogares de la ciudad, es el agua lluvia. Como se mencionó en la introducción, son bastantes los países que en la actualidad cuentan con normativas claras que exigen tanto a arquitectos y urbanistas, como a los propios usuarios, el uso del recurso pluvial con tres fines básicos: descarga de sanitarios, riego de jardines y lavado de automóviles, y eventualmente, lavado de ropa.

En este orden de ideas, se realizaron estimativos a futuro referidos a las ventajas de aprovechar el recurso pluvial en Bogotá con el objetivo de obtener dos datos: la cantidad de metros cúbicos de agua potable que dejarían de ser suministrados por el acueducto de Bogotá, y el valor económico que ese ahorro en el consumo de agua -proveniente del acueducto- representaría para la ciudadanía. Para elaborar los cálculos se tomaron datos del Catastro Distrital (2015) correspondientes a número total de viviendas de Bogotá; porcentaje de viviendas para estratos 1 , 2, 3, 4, 5 y 6; porcentaje de casas, porcentaje de apartamentos y porcentaje de establecimientos no residenciales. A partir de los datos obtenidos del Catastro Distrital, se estableció el ahorro (en metros cúbicos y pesos colombianos de 2015) si se sustituye el agua del acueducto por agua lluvia en un $10 \%$ del consumo promedio actual, en el 10\% de las viviendas tipo casa y en el 10\% de los establecimientos no residenciales. Bajo estos parámetros que se deben subrayar, que son bastante conservadores, se concluyó que el ahorro en 2015 sería de más de un millón y medio de metros cúbicos de agua potable; y el ahorro en dinero equivaldría a casi siete mil millones de pesos colombianos (de 2015). Para 2035, estas cifras alcanzarían hasta 2.284.240 de metros cúbicos de ahorro de agua, y algo más de diez mil millones de pesos (de 2015). Al sumar los ahorros que se alcanzarían durante el período de veinte años establecido para elaborar los estimativos (2015-2035), las cifras son astronómicas: en agua se dejarían de consumir 40,1 millones de metros cúbicos del acueducto, y por concepto de ahorro en los recibos de pago, los habitantes de Bogotá ahorrarían \$177.516 millones de pesos colombianos (de 2015).

Si bien estos estimativos aportan claridad sobre los enormes beneficios de gestionar las aguas pluviales en Bogotá, sus objetivos se enfocan en los usuarios y se deja de lado otros enormes costos, es decir, los que devienen de la cadena de valor del acueducto, que en Colombia incluye: 1 . captación de agua cruda; 2 . conducción de agua cruda (aducción); 3. tratamiento de agua cruda; 4. conducción de agua potable; 5 . distribución de agua potable; y 6. comercialización. ¿Cuánto le costará al Distrito la cadena de valor del acueducto correspondiente a esos 40,1 millones de metros cúbicos de agua que podrían obviarse si se aprovecha el recurso pluvial? Así mismo, cabe una consideración económica final: una política clara en cuanto a la gestión del agua lluvia en Bogotá tendrá un tercer y muy importante efecto, la reducción de inversiones futuras en las infraestructuras necesarias para ensanchar las redes de abastecimiento del acueducto en la ciudad. 


\section{Conclusiones y recomendaciones}

Luego de revisar e interpretar los resultados obtenidos por la investigación, se concluye que, en Colombia, la planificación territorial y el diseño urbano deben tener en cuenta la lluvia como determinante fundamental para la construcción del paisaje y la recreación urbana, como componente clave para la conservación de los ecosistemas y la biodiversidad en ambientes urbanos y periurbanos, como patrimonio ciudadano, como elemento ordenador en centros urbanos experimentales o innovadores y como reductor del consumo de agua potable en las ciudades. A nivel arquitectónico, es evidente que la implementación del acopio, almacenamiento y uso del agua lluvia en las edificaciones de Bogotá es casi inexistente, y lo mismo sucede en el resto del país. Es prioritario, entonces, que las edificaciones respondan de manera eficiente y responsable a la gestión del recurso pluvial, y para ello se requiere que durante el proceso de diseño, los arquitectos tengan en cuenta los sistemas de captación, almacenamiento, distribución y aprovechamiento de las aguas lluvias. En el escenario económico cabe subrayar que, teniendo en cuenta los importantes ahorros en agua potable y dinero que pueden derivarse del aprovechamiento del recurso pluvial en las ciudades, resulta estratégico y urgente adelantar un programa a nivel nacional que ayude a difundir, promover e incentivar el uso de sistemas para la recolección y uso del agua lluvia en el medio urbano, y que contribuya a suavizar las crisis de agua que podrían enfrentar las ciudades colombianas en un futuro cada vez más cercano.

Algunas universidades ya están trabajando en esos temas, pero resulta imprescindible que los resultados de investigación que surgen de la academia permeen prontamente las políticas públicas y la normativa urbana de las ciudades y los municipios colombianos; y sean tenidos en cuenta y asimilados por los arquitectos, urbanistas, planificadores y funcionarios públicos que tienen en sus manos la responsabilidad de diseñar y planificar las edificaciones y las ciudades colombianas del siglo XXI, ya que, por la seguridad de todos sus habitantes, estas deben ser sustentables y resilientes. La gestión estratégica del recurso pluvial es pieza fundamental para alcanzar estos objetivos.

Por último, se recomienda promover el uso del recurso pluvial en Colombia con el fin de: i) incrementar la descentralización de las redes de acueducto por parte de la ciudadanía, lo que estimularía la autonomía hídrica; ii) reducir el consumo del agua potable que circula por los acueductos urbanos, al destinar su uso principalmente al consumo humano; iii) incrementar la resiliencia urbana al contar con agua, aunque se interrumpa el abastecimiento proveniente del acueducto; iv) reducir la presión sobre los ecosistemas a los que se le 'secuestra' el agua para llevarla a las ciudades, puesto que se aprovecha el agua lluvia; v) minimizar los costos y volúmenes de acopio, potabilización y trasporte del agua para los acueductos; vi) rebajar el costo de las facturas de acueducto que deben pagar los ciudadanos; y vii) minimizar los riesgos de inundación en áreas urbanas.

\section{Referencias}

Aalborg Kommune. (2013). Baredygtighedsstrategi. Aalborg Kommune: Sundhed og Bæredygtig Udvikling. Recuperado de https://www. aalborg.dk/media/271693/baeredygtighedsstrategi-2013-16.pdf.

Adger, W. N. (2006). Vulnerability. Global Environmental Change, 16(3), 268-281.

Aladenola, O. O., y Adeboye, O. B. (2010). Assessing the potential for rainwater harvesting. $\mathrm{Wa}$ ter Resources Management, 24(10), 2129-2137.

Alcaldía Mayor de Bogotá. (2014, 24 de noviembre). Decreto 528 de 24 de noviembre de 2014. Recuperado de http://oab2.ambientebogota.gov.co/ 
es/documentacion-e-investigaciones/resultadobusqueda/decreto-528-de-24-de-noviembre -de-2014.

Allerød Kommune. (2013). Grundvandsstrategi. Allerød: Allerød Kommune. Recuperado de http://alleroed-kp17.cowi.webhouse.dk/download/grundvandsstrategi.pdf.

Andersen, L., y Steffensen, A. (2011). Baredygtig Håndtering af Regnvand. Roskilde, Dinamarca: Roskilde Universitet.

Annecchini, K. P. (2005). Aproveitamento da água da chuva para fins não potáveis na cidade de Vitória (ES) (tesis de posgrado). Universidade Federal do Espírito Santo, Vitoria, Brasil.

Bacchin, T. K., Ashley, R., Sijmons, D., Zevenbergen, C., y Timmeren, A. (2014, septiembre). Green-Blue Multifunctional Infrastructure: An Urban Landscape System Design New Approach. Ponencia presentada en $13^{\text {th }}$ International Conference on Urban Drainage, Sarawak.

Bloch, R. (2003). Los conflictos por el agua dulce en el mundo. Buenos Aires: Editorial Duplicar.

Boroomand, L. (2015). NEWater urban scapes. A methodology for assessing water sensitive cities (tesis de maestría). Politécnico di Milano, Milán, Italia.

Botkyrka Kommun. (2012). Dagvattenstrategi för Botkyrka Kommun. Botkyrka Kommun: kommunfullmäktige. Recuperado de https:// www.botkyrka.se/download/18.4a23abd9158495687c9ebc80/1486981440077/Dagvattenstrategi.pdf.

Buhaug, H., y Urdal, H. (2013). An urbanization bomb? Population growth and social disorder in cities. Global Environmental Change, 23(1), 1-10.

Buitrago-Medina, N. F. (2011). Cuantificación y caracterización de la calidad de agua de escorrentía de techo para el prediseño de una piscina de retención en el campus de la Universidad Nacional de Colombia (tesis doctoral). Universidad Nacional de Colombia, Bogotá, Colombia.

BUP Bruxelles Urbanisme et Patrimoine. (2006). Règlement Régional d'Urbanisme. Bruselas: Ministère de la région de Bruxelles-Capitale.

Carvajal-Escobar, Y. (2011). Inundaciones en Colombia. ¿Estamos preparados para enfrentar la variabilidad y el cambio climático? Revista Memorias, 9(16), 105-119.

Catastro Distrital. (2015). Resultados censo inmobiliario 2015. Bogotá: Unidad Administrativa Especial de Catastro Distrital (UAECD). Recuperado de https://www.catastrobogota.gov. $\mathrm{co} /$ sites/default/files/PresentacionCensoInmobiliario2015.pdf

Conseil Fédéral Suisse. (2014). Ordonnance sur la protection des eaux-OEaux. Ginebra: Conseil Fédéral Suisse. Recuperado de https://www. micropoll.ch/fileadmin/user_upload/Redaktion/Dokumente/04_Gesetzliche_Grundlagen/ Ordonnance_sur_la_protection_des_eaux_. OEaux___Projet_du_22_d\%C3\%A9cembre_ 2014.pdf.

Departamento de Asuntos Económicos y Sociales de Naciones Unidas (ONU-DAES). (2015). Decenio internacional para la acción "El agua fuente de vida" 2005-2015. Recuperado de https://www. un.org/spanish/waterforlifedecade/.

Departamento Técnico Administrativo del Medio Ambiente (DAMA). (2000). Historia de los humedales de Bogotá: con énfasis en cinco de ellos. Bogotá: DAMA.

Environnement et Lutte contre les changements climatiques Québec. (2014). Guide de gestion des eaux pluviales. Recuperado de http://environnement.gouv.qc.ca/eau/pluviales/guide-gestion-eaux-pluviales.pdf 
Estupiñán-Perdomo, J. L., y Zapata-García, H. O. (2010). Requerimientos de infraestructura para el aprovechamiento sostenible del agua lluvia en el campus de la Pontificia Universidad Javeriana, sede Bogotá (tesis de maestría). Pontificia Universidad Javeriana, Bogotá, Colombia.

Falu Kommun. (2008). Dagvattenstrategi för Falu kommun. Falu kommun: Falu Energi \& Vatten AB, Stadsbyggnadskontoret Trafik- och Fritidsförvaltningen. Recuperado de http://www2.falun. se/www/governing.nsf/doc/EAD007112116A0EFC12582970059D0E5/\$File/Dagvattenstrategi_ Falun.pdf?OpenElement.

Fernández-Pérez, I. (2009). Aprovechamiento de aguas pluviales. Barcelona: Escola Politècnica Superior d'edificació de Barcelona.

Furesø Kommune. (2010). Strategi for Håndtering af Regnvand. Furesø Kommune: Byrådet. Recuperado de https://www.furesoe.dk/media/2384/strategi-for-haandtering-af-regnvand.pdf.

Gentofte Kommune. (2010). Klimatilpasnings-strategi for Gentofte Kommune. Gentofte Kommune: Teknik og Miljø.

Gleick, P. H. (2010). Water, War y Peace in Middle East. Environment: Science and Policy for Sustainable Development, 36(3), 6-42.

Gómez, A., Galarza-Molina, S., y Torres, A. (2018). Propuesta de mejoramiento tecnológico de techos verdes para el clima tropical andino. Ciencia e Ingeniería Neogranadina, 28(1), 73-99.

Göransson, S. (2013). En ny mötesplats i Lövholmen, Stockholm. Uppsala: Sveriges lantbruksuniversitet.

Grand Lyon. (2010). Les ouvrages aériens de gestion des eaux pluviales. Référentiel conception et gestion des espaces publics. Lyon: Direction de l'Eau. Recuperado de https://www.grandlyon. com/fileadmin/user_upload/media/pdf/voirie/ referentiel-espaces-publics/20091201_gl_refe- rentiel_espaces_publics_ouvrages_aeriens_gestion_eaux_pluviales.pdf.

Grand Lyon. (2013). La gestion intégrée des eaux pluviales. Nature et bien-être en ville: Des solutions adaptées au changement climatique. Lyon: Direction de l'Eau. Recuperado de https://www. grandlyon.com/fileadmin/user_upload/media/ pdf/eau/assainissement/20130530_gl_gestion-integree-eauxpluviales.pdf

Härryda Kommun. (2011). Gällande Dagvattenpolicy i Härryda Kommun. Härryda Kommun: Sweco. Recuperado de https://www.harryda. se/download/18.43b9a916300754f9e85db6/15 25423953759/Bilaga\%203\%20-\%20Dagvattenpolicy.pdf.

Huddinge Kommun. (2000). Huddinge Kommun Dagvattenstrategi. Huddinge: Huddinge Kommun / Stockholm Vatten. Recuperado de http:// miljobarometern.huddinge.se/content/docs/Dagvattenstrategi $\% 202000$.pdf.

Hvidovre Kommune. (2014). Strategi for Klimatilpasning 2014. Hvidovre Kommune: Byrådet.

Institut Bruxellois pour la Gestion de l'Environnement (IBGE). (2010). Gerer les eaux pluviales sur la parcelle. Bruselas: IBGE. Recuperado de https://forest.irisnet.be/fr/services-communaux/ developpement-urbain/fichiers/info-fiche-gestion-sur-la-parcelle.pdf

Instituto de Hidrología, Meteorología y Estudios Ambientales (IDEAM). (2010). Estudio Nacional del Agua. Bogotá: IDEAM. Recuperado de http://www.andi.com.co/Uploads/ENA_2010. compressed.pdf

Instituto de Hidrología, Meteorología y Estudios Ambientales (IDEAM). (2014). Estudio Nacional del Agua. Bogotá: IDEAM. Recuperado de http://documentacion.ideam.gov.co/openbiblio/ bvirtual/023080/ENA_2014.pdf 
Kim, H., Kwack, D., Yoon, M., Lee, J., y Hwang, Y. (2014). Residential Environment Function and Evaluation of Water Sensitive Urban Design. Journal of the Residential Environment Institute of Korea, 12(3), 327-341.

Københavns. (2011). Københavns Klimatilpasningsplan. Københavns: Københavns Kommune.

Kruse, T. (2005). La guerra del agua en Cochabamba, Bolivia: terrenos complejos, convergencias nuevas. En E. De la Garza-Toledo (comp.), Sindicatos y nuevos movimientos sociales en América latina (pp. 121-161). Buenos Aires, Argentina: Clacso.

Kungsbacka Kommun. (2012). Dagvatten Policy och Riktlinjer. Kungsbacka Kommun: Förvaltningen för Teknik. Recuperado de https://www. kungsbacka.se/globalassets/kommun-och-politik/dokument/styrdokument/policy/dagvatten_-feb2013_webb.pdf.

Legifrance. (2014). Code général des collectivités territoriales. Disponible en: http://www. legifrance.gouv.fr/affichCode.do;jsessionid=AD5147F4771982808F78CE92682D03AF.tpdjo04v_2?cidTexte=LEGITEXT000006070633 \&dateTexte $=20141105$

Masthusen. (2013). Dagvattenhantering i Masthusen. Masthusen: Sweco. Recuperado de http:// www.masthusen.se/contentassets/ecec $5 \mathrm{daa}-$ 106d42a89e911f3d0a95f596/2.-dagvattenhantering-i-masthusen-131207.pdf.

Mendez, C. B., Bae, S., Chambers, B., Fakhreddine, S., Gloyna, T., Keithley, S., ... Kirisits, M. J. (2010). Effect of roof material on water quality for rainwater harvesting systems. Austin: Texas Water Development Board.

Miljøministeriet. (2014). Brug af regnvandsanleg i Danmark. Københavns: Miljøministeriet.
Recuperado de https://naturstyrelsen.dk/media/ nst/7496658/140304\%20Brug\%20af\%20regnvandsanl\%C3\%A6g\%20i\%20Danmark_erfaringsopsamtling_FINAL\%20(2).pdf.

Molina-Prieto, L. F. (2014). Gestión urbana del recurso pluvial: aproximación histórica. Revista de Investigación, 7(2), 174-187.

Molina-Prieto, L. F. (2015). Gestión urbana del recurso pluvial: estrategias, políticas y normativa urbana en cinco países europeos. Revista de Investigación, 8(1), 125-138.

Molina-Prieto, L. F. (2016). Resiliencia a inundaciones: nuevo paradigma para el diseño urbano. Revista de Arquitectura, 18(2), 82-93.

Morgan, C., Bevington, C., Levin, D., Robinson, P., Davis, P., Abbott, J., y Simkins, P. (2013). Water Sensitive Urban Design in the UK-Ideas for built environment practitioners. Londres: CIRIA.

Nacka Kommun. (2011). Anvisningar för Dagvattenhantering i Nacka Kommun. Nacka Kommun: Kommunstyrelsen. Recuperado de https://www. nacka.se/4adbfd/globalassets/stadsutveckling-trafik/dokument/stadsutveckling/markanvisning/ kraftledningsstraket/bilaga_13_anvisningar_dagvattenhantering_nacka.pdf.

Naturstyrelsen. (2012/2013). Sådan håndterer vi skybrud og regnvand. Handlingsplan for klimasikring af Danmark. København: Naturstyrelsen/Task Force for Klimatilpasning. Recuperado de https://naturstyrelsen.dk/media/nst/Attachments/Klimahandlingsplan_dec_2012.pdf

Naturvårdsverket. (2019). Regeringsuppdrag att föreslå etappmål om dagvatten. Stockholm: $\mathrm{Na}$ turvårdsverket. Recuperado de https://www.naturvardsverket.se/upload/miljoarbete-i-samhallet/ miljoarbete-i-sverige/regeringsuppdrag/2019/redovisning-ru-etappmal-for-dagvatten-skrivelse.pdf. 
Nelson, V., Moddemeyer, S., y Schwartz, P. (2007). The Baltimore Charter for Sustainable Water Systems. Recuperado de http://sustainablewaterforum.org/baltimore.html.

Odense Kommune. (2011). Klimatilpasning $i$ Odense Kommune. Odense Kommune: Byrådet. Recuperado de https://www.klimatilpasning.dk/ media/359549/charlottemoosdorf.pdf

Office cantonal de l'eau (OCEau). (2015). Formularie: Traitement des eaux de chantier. Cantón de Ginebra: Office cantonal de l'eau (OCEau), OCEau - SECOE - Secteur protection des eaux.

Orozco-Roa, P. A. (2016). Alternativas para el manejo de aguas pluviales en medios urbanos. Estudio de caso: implementación y manejo de los canales pluviales en las cuencas del Salitre y Tintal en el marco del proceso de recuperación Río Bogotá 2000-2014 (tesis de pregrado). Universidad Colegio Mayor de Nuestra Señora del Rosario, Bogotá, Colombia.

Österåkers Kommun. (2010). Dagvattenstrategi - en vägledning och handbok för dagvattenhantering inom Österåkers kommun. Österåkers Kommun: Sweco. Recuperado de https://www. osteraker.se/download/18.1f86c0e614421c414cealf9/1446720544565/Dagvattenstrategi_ for_Osteraker.pdf.

Perló-Cohen, M., y González-Reynoso, A. E. (2009). ¿Guerra por el agua en el valle de México? Estudio sobre las relaciones hidráulicas entre el Distrito Federal y el Estado de México. México D.F.: UNAM.

Philadelphia Water Department. (2014). City of Philadelphia Green Streets Design Manual. Recuperado de http://www.phillywatersheds.org/img/ GSDM/GSDM_FINAL_20140211.pdf.

Polanco-Andrade, A. M., y Sánchez-Vega, A. M. (2012). Diseño hidráulico de losas en pavimento poroso rígido como estructuras complementarias al drenaje pluvial de Bogotá (tesis de pregrado). Pontificia Universidad Javeriana, Bogotá, Colombia.

Ranger, N., Hallegatte, S., Bhattacharya, S., Bachu, M., Priya, S., Dhore, K., ... Corfee-Morlot, J. (2011). An assessment of the potential impact of climate change on flood risk in Mumbai. Climatic Change, (104), 139-167.

Ringsted Kommune. (2011). Klimaplan for Ringsted Kommune. Ringsted Kommune: Byrådet. Recuperado de https://ringsted.dk/sites/ default/files/acadre/mm/Files_1217_1010186/ klimaplan.pdf.

Roskilde Kommune. (2013). Vand \& Klimatilpasning Strategi. Roskilde Kommune: Kulturog Miljø.

Sandoval, G. (2016). Ventajas económicas del aprovechamiento del agua lluvia. Equidad \& Desarrollo, (26), 101-113.

Secretaría Distrital de Ambiente (SDA). (2011). Resolución 6523 de 2011 "Por la cual se reglamentan y adoptan los sistemas urbanos de drenaje sostenibles SUSD para el plan de ordenamiento zonal norte POZN". Bogotá: SDA.

Secretaría Distrital de Ambiente (SDA). (2014). Techos Verdes y Jardines Verticales, guía práctica. Bogotá: SDA. Recuperado de http://ambientebogota.gov.co/es/web/publicaciones-sda/ techos-verdes-y-jardines-verticales 1 .

Service de la planification de l'eau. (2009). Gestion des eaux non polluées à la parcelle - Aspects quantitatifs. Cantón de Ginebra: Service de la planification de l'eau.

Shiva, V. (2004). Le guerre dell'acqua. Milán: Giangiacomo Feltrinelli Editore.

Site officiel de l'Etat de Genève. (2014). Loi sur les eaux (LEaux-GE). Recuperado de http://www. ge.ch/legislation/rsg/f/s/rsg_12_05.html 
Skåne Län. (2009). PlanPM Dagvatten. Skåne Län: Länsstyrelsen i Skåne Län. Recuperado de https://www.lansstyrelsen.se/download/18.26f50 6e0167c605d569477d5/1551710360972/PM_ dagvatten.pdf.

Stockholms Stad. (2001). Ta hand om ditt vatten. Stockholms Stad: Stockholm Vatten. Recuperado de http://miljobarometern.huddinge.se/ content/docs/Ta\%20hand $\% 20$ om $\% 20 \mathrm{ditt} \% 20$ vatten[1].pdf.

Stockholms Stad. (2013). Dagvattenstrategi Stockholms väg till en hållbar dagvattenhantering. Stockholm: Trafikkontoret, Miljöförvaltningen, Stadsbyggnadskontoret, Exploateringskontoret, Stockholm Vatten. Recuperado de https://stad. stockholm/globalassets/om-stockholms-stad/ politik-och-demokrati/styrande-dokument/dagvattenstrategi-stockholms-vag-till-en-hallbar-dagvattenhantering.pdf.

Structor. (2016). Dagvattenutredning- Weibullsholm, Landskrona. Malmö: Structor. Recuperado de https://www.landskrona.se/globalassets/ invanare/stadsplanering--trafik/detaljplaner/pagaende-planarbeten/orja-347/va-utredning_weibullsholm_161117.pdf.

Tello-Aragay, E. (1998). La "guerra del agua" en Barcelona. Alternativas económico-ecológicas para un desafío socioambiental. Mientras tanto, (73), 55-72.

Torres, A., Méndez-Fajardo, S., Lara-Borrero, J. A., Estupiñán-Perdomo, J. L., Zapata-García, H. O., y Torres-Murillo, Ó. M. (2012). Hacia equipamientos urbanos sostenibles: aprovechamiento de aguas lluvias en el campus de la Pontificia Universidad Javeriana en Bogotá. Cuadernos de Vivienda y Urbanismo, 5(9), 124-141.
Torres, A., Méndez-Fajardo, S., López-Kleine, L., Marín, V., González, J. A., Suárez, J. C., ... Ruiz, A. (2011). Evaluación preliminar de la calidad de la escorrentía pluvial sobre tejados para su posible aprovechamiento en zonas periurbanas de Bogotá. UDCA Actualidad y divulgación cientifica, 14(1), 127-135.

Torres-Murillo, O. M. (2011). Identificación de los posibles usos del agua lluvia de escorrentia en el campus de la Pontificia Universidad Javeriana (Bogotá) (tesis de pregrado). Pontificia Universidad Javeriana, Bogotá, Colombia.

Trafikverket. (2011). Vägdagvatten - Råd och rekommendationer för val av miljöåtgärd. Borlänge: Trafikverket. Recuperado de https:// trafikverket.ineko.se/Files/sv-SE/11439/RelatedFiles/2011_112_vagdagvatten_rad_och_rekommendationer_for_val_av_miljoatgard.pdf

Tyresö kommun. (2010). Riktlinjer för dagvattenhantering $i$ Tyresö kommun. Tyresö kommun: Sweco. Recuperado de https://insynsverige.se/ documentHandler.ashx?did=1777798.

Värmdö kommun. (2012). Dagvattenpolicy för Värmdö kommun. Värmdö kommun: Renhållningsenheten. Recuperado de https://www. varmdo.se/download/18.22e4ee451639ed6e2d8af1b3/1528124585307/Dagvattenpolicy\%20 f\%C3\%B6r\%20V\%C3\%A4rmd\%C3\%B6\%20 kommun.pdf.

Wilby, R., y Keenan, R. (2012). Adapting to flood risk under climate change. Progress in Physical Geography, 36(3), 1-31.

Wong, T. H. F., Allen, R., Brown, R. R., Deletic, A., Gangadharan, L., Gernjak, W., ... Walsh, C. J. (2013). Blueprint 2013 - Stormwater Management in a Water Sensitive City. Melbourne: Cooperative Research Centre for Water Sensitive Cities. 
1 En las ciudades actuales un gran porcentaje del agua potable se destina a lavado de carros, riego de jardines y descarga de sanitarios.

2 En Bogotá, por ejemplo, solamente por concepto de descargas de sanitarios se arrojan al sistema de alcantarillado cerca de 87,6 millones de metros cúbicos de agua por año, volumen comparable al que almacena el embalse del Sisga en sus 700 hectáreas de superficie (Molina-Prieto, 2014).

3 Se estima que, por cuenta del desarrollo urbano, el sistema de lagos y humedales de la Sabana de Bogotá pasó de contar con 55.000 hectáreas en 1920 a 800 en el año 2000 (DAMA, 2000)

4 Acopio de agua lluvia. Sustentabilidad. Reducir índice de impermeabilización del suelo. Suprimir escorrentías e inundaciones. Resiliencia urbana. Gestión de agua pluvial in situ. Evitar recarga de alcantarillados y plantas de tratamiento. Fortalecer ciclo del agua. Mantener nivel freático. Fomentar techos verdes y sistemas de biorretención. Limitar uso de agua para la construcción.

5 Bajo este enfoque se establecen las políticas nacionales del agua en el Código Nacional de Recursos Naturales y de Protección del Medio Ambiente (Decreto 2811 de 1974); en la Ley 142 de 1994; en los artículos 1, 334, 365, 366 y 370 de la Constitución Política de Colombia; y en la suscrita Cumbre del Milenio ratificada por el Compes Social 91 de 2005; y las políticas distritales compendiadas en el artículo 77 del Plan de Ordenamiento Territorial POT; el Plan Maestro de Acueducto y Alcantarillado para Bogotá Distrito Capital; el Decreto Distrital 456 de 2008; el Acuerdo 347 del 23 de diciembre de 2008; el Plan Distrital del Agua y el Decreto 485 de 2011 que lo adopta.

6 Estándar de las Naciones Unidas, con el cual se valora la presión de la demanda sobre la oferta hídrica en regiones establecidas.

7 Usado para "medir la capacidad de retención de humedad de las cuencas con base en la distribución de las series de frecuencias acumuladas de los caudales diarios" (Ideam, 2010, p. 323).

8 "grado de fragilidad del sistema hídrico para mantener una oferta para el abastecimiento de agua, que ante amenazas -como periodos largos de estiaje 0 eventos como el Fenómeno cálido del Pacífico (El Niño)- podría generar riesgos de desabastecimiento" (Ideam, 2010, p. 329). 